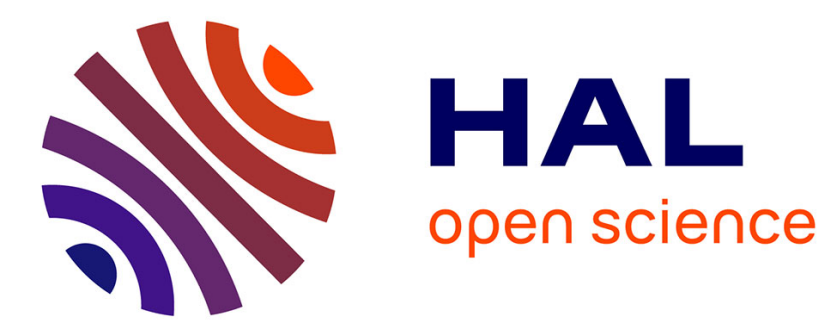

\title{
The Finiteness of Determining Degrees of Freedom for the Quasi-Geostrophic Multi-Layer Ocean Model
}

Christine Bernier-Kazantsev, Igor D. Chueshov

\section{To cite this version:}

Christine Bernier-Kazantsev, Igor D. Chueshov. The Finiteness of Determining Degrees of Freedom for the Quasi-Geostrophic Multi-Layer Ocean Model. [Research Report] RR-3067, INRIA. 1996, pp.17. inria-00073625

\section{HAL Id: inria-00073625 \\ https://hal.inria.fr/inria-00073625}

Submitted on 24 May 2006

HAL is a multi-disciplinary open access archive for the deposit and dissemination of scientific research documents, whether they are published or not. The documents may come from teaching and research institutions in France or abroad, or from public or private research centers.
L'archive ouverte pluridisciplinaire HAL, est destinée au dépôt et à la diffusion de documents scientifiques de niveau recherche, publiés ou non, émanant des établissements d'enseignement et de recherche français ou étrangers, des laboratoires publics ou privés. 


\section{The finiteness of determining degrees of freedom for the quasi-geostrophic multi-layer ocean model.}

Christine Bernier-Kazantsev, Igor D. Chueshov

\section{$\mathbf{N}^{\circ} 3067$}

Decembre 1996

THÈME 4 



\title{
RINRIA
}

\section{The finiteness of determining degrees of freedom for the quasi-geostrophic multi-layer ocean model.}

\author{
Christine Bernier-Kazantsev * ${ }^{*}$ Igor D. Chueshov ${ }^{\dagger}$ \\ Thème 4 - Simulation et optimisation \\ de systèmes complexes \\ Projet NUMATH \\ Rapport de recherche $\mathrm{n}^{\circ} 3067$ - Decembre 1996 - 17 pages
}

\begin{abstract}
In this paper, we consider a multi-layer quasi-geostrophic model of the ocean dynamics and we prove that the long-time behaviour of its solutions can be described by a finite number of determining parameters. Under some additional condition we also show that the dynamics of the bottom layer is completely determined by parameters connected with upper layers only. It means that the information about the bottom layer is not essential for a description of the long-time behaviour of the system under consideration.
\end{abstract}

Key-words: Determining degree of freedom, quasi-geostrophic model.

(Résumé : tsvp)

\footnotetext{
* Institut Elie Cartan, CNRS UMR 9973, INRIA-Lorraine, Université H. Poincaré-Nancy 1, BP 239, 54506 Vandoeuvre-les-Nancy Cedex, France.

† Dept. of Mechanics and Mathematics, Kharkov University, 4 Svobody sq., Kharkov 310077 , Ukraine.
} 


\section{Nombre fini de degrés de liberté déterminants pour un modèle quasi-géostrophique multicouches de l'océan.}

Résumé : Dans ce travail, on considère un modèle quasi-géostrophique multicouche de l'océan et on démontre que le comportement asymptotique de ses solutions peut être décrit par un nombre fini de paramètres déterminants. Sous certaines conditions supplémentaires, on montre également que la dynamique de la couche de fond est complètement déterminée par des paramètres connectés seulement aux couches supérieures. Ceci veut dire que l'information sur la couche de fond n'est pas essentielle pour décrire le comportement asymptotique du système que l'on considère.

Mots-clé : Degrés de liberté déterminants, modèle quasi-géostrophique. 


\section{Introduction.}

Ocean circulation numerical modelling started in the sixties with Sarkisyan [23] and Bryan and Cox [3]. Still now, this problem remains under consideration and recently Lions, Temam and Wang proposed a modelisation for large scale ocean and atmosphere dynamics and developed a mathematical theory for the coupled atmosphere-ocean models [21][22]. The equations of the velocity are derived from the Navier-Stokes equations, with adjonction of the Coriolis' effect. The behaviour of temperature and salinity or density are described also by partial differential equations. Due to the complexity of the problem, simpler models were introduced. The quasi-geostrophic (QG) one is a simple formulation neglecting thermodynamics effects. First, the density is supposed to be constant and the equations are integrated on the vertical. This results in a two-dimensional Navier-Stokes equation with the Coriolis' effect. The QG model corresponds to the streamfunction-vorticity formulation of this equation. In fact, assuming the density to be constant in the ocean is a rough approximation. Thus in order to improve the model, Holland [16] introduced a two-layer formulation of this model, assuming the density is constant in each layer. The QG equation is written in each layer with a suitable coupling through convection terms. More recently similar models with K-layers have been introduced [20]. In this paper we consider the K-layers QG formulation of the ocean dynamics. We are first interested in which parameters can be used for the description of the long-time behaviour of the solutions to the problem (1)-(7), and second in how many parameters are necessary for this description. These questions are inspired by the paper of Foias-Prodi [13] about determining modes for the 2D Navier-Stokes equations and are extensively discussed in the literature (see, e.g. [5], [6], [7], [9],[10],[12]$[15],[17],[18])$. The concepts of determining nodes and determining local volume averages were introduced in [14] and [15] [17] respectively. A general approach to the problem of the existence of a finite number of determining parameters (degrees of freedom) was discussed in [7], [9], [10].

\section{Model equations and preliminaries.}

We consider ocean dynamics in the quasi geostrophic formulation, i.e. all thermodynamic effects are neglected. The vertical structure of the ocean is modelled by splitting the domain $\Omega$ into $K$ layers of depth thickness $H_{k}$. The equation of the

$\operatorname{RR} \mathrm{n}^{\circ} 3067$ 
dynamics then reads $[16,20]$

$$
\begin{aligned}
\frac{\partial \theta_{k}}{\partial t}+J\left(\psi_{k}, \theta_{k}+\beta y\right)=\mu \Delta^{2} \psi_{k}+F_{k}^{\text {wind }}-D_{k}^{\text {bottom }} & \text { in } \Omega, \quad k=1, \ldots, K, \\
\theta=\Delta \psi-\mathcal{W} \psi & \text { in } \Omega
\end{aligned}
$$

where $\psi=\psi_{k}(x, y), k=1, \ldots, K$ is the quasi geostrophic streamfunction of the $k$-th layer. We suppose that $\Omega$ is a bounded open subset of $\mathbb{R}^{2}$ with smooth boundary $\partial \Omega$. The $k^{t h}$ layer is characterized by its thickness $H_{k}$, its reduced gravity $g_{k}$ and its streamfunction $\psi_{k}$. The forcing $F_{k}^{\text {wind }}$ infers only on the upper layer: $F_{k}^{\text {wind }}=0$ for $k \neq 1$ and $F_{1}^{\text {wind }}=v / H_{1}$, where $v$ is equal to the curl of the wind stress on the surface and is supposed to be in $L^{2}(\Omega)$. The bottom drag is $D_{k}^{\text {bottom }}=0$ for $k \neq K$ and $D_{K}^{b o t t o m}=\sigma \Delta \psi_{K}$. We consider the linear approximation only of the parameter of Coriolis $f=f_{0}+\beta y$ where $f_{0}$ and $\beta$ are the parameter of Coriolis and its meridional gradient in the middle of the bassin. The Jacobian operator is defined by

$$
J(u, v)=\frac{\partial u}{\partial x} \frac{\partial v}{\partial y}-\frac{\partial u}{\partial y} \frac{\partial v}{\partial x} .
$$

The matrix $\mathcal{W}$ is the $K \times K$ tridiagonal matrix defined by

$$
\mathcal{W}=\left(\begin{array}{cccc}
R_{1} & -R_{1} & \cdots & 0 \\
-R_{2}^{\prime} & R_{2}+R_{2}^{\prime} & -R_{2} & \cdots \\
\vdots & \ddots & \ddots & \ddots \\
0 & \cdots & -R_{K}^{\prime} & R_{K}^{\prime}
\end{array}\right) \text { where }\left\{\begin{array}{c}
R_{k}=\frac{f_{0}^{2} \rho_{0}}{g H_{k}\left(\rho_{k+1}-\rho_{k}\right)} \\
R_{k}^{\prime}=\frac{f_{0}^{2} \rho_{0}}{g H_{k}\left(\rho_{k}-\rho_{k-1}\right)}
\end{array}\right.
$$

where $\rho_{0}$ is the mean density of water, $\rho_{k}$ is the mean density in the $k$-th layer and $g$ is the gravity acceleration.

We notice that the matrix $\mathcal{W}$ has a complete system of eigenvectors. We thus introduce $\Lambda$ the non-negative diagonal matrix of the eigenvalues of $\mathcal{W}$, and define the matrix $B$ so that $\Lambda=B^{-1} \mathcal{W} B$. We shall denote each vector in the eigenbasis of $\mathcal{W}$ by a star:

$$
\psi^{*}=B^{-1} \psi, \theta^{*}=B^{-1} \theta, \text { etc. }
$$


We supplement (1) and (2) with the following boundary conditions :

$$
\left\{\begin{array}{lll}
\theta(x, y, t)+\mathcal{W} \psi(x, y, t)=0 & \text { on } & \partial \Omega \times] 0, T[, k=1, \ldots, K \\
\psi_{k}^{*}(x, y, t)=C_{k}^{*}(t) & \text { on } & \partial \Omega \times] 0, T[, k=1, \ldots, K \\
\int_{\Omega} \psi_{k}^{*} d \omega=0, & k=2, \ldots, K, \\
C_{1}^{*}=0 . & &
\end{array}\right.
$$

Let us point out here that $\left(C_{k}^{*}(t)\right)_{2 \leq k \leq K}$ are unknown functions. Finally, the initial data is

$$
\theta_{k}(x, y, 0)=\theta_{0, k}(x, y) \text { in } \Omega, k=1, \ldots, K,
$$

where $\theta_{0, k}(x, y)$ is in $H^{-1}(\Omega), k=1, \ldots, K$. The unknown functions are $\psi_{k}(x, y, t)$, $\theta_{k}(x, y, t)$ and $C_{k}^{*}(t)$. From now on, we denote by $\mathbf{H}^{\mathbf{- 1}}, \mathbf{L}^{\mathbf{2}}, \mathbf{H}^{\mathbf{1}}$, and so on the spaces $\left(H^{-1}(\Omega)\right)^{K},\left(L^{2}(\Omega)\right)^{K},\left(H^{1}(\Omega)\right)^{K}$ etc. We also introduce the constant $p_{k}, k=$ $0, \ldots, K$ defined by $p_{0}=0, p_{K}=0, p_{k}=\frac{f_{0}^{2} \rho_{0}}{g\left(\rho_{k+1}-\rho_{k}\right)}=H_{k} R_{k}=H_{k+1} R_{k+1}^{\prime}, k=$ $1, \ldots, K-1$. As in [2], we will use the norm $\|\cdot\|_{-1}$ on $\mathbf{H}^{-1}$ defined by the scalar product

$$
((\theta, \bar{\theta}))=\sum_{1}^{K} H_{k} \int_{\Omega} \nabla \psi_{k} \cdot \nabla \bar{\psi}_{k}+\sum_{i}^{K} p_{k} \int\left(\psi_{k}-\psi_{k+1}\right) \cdot\left(\bar{\psi}_{k}-\psi_{k+1}^{-}\right)
$$

where $\psi($ resp. $\bar{\psi}$ ) are defined by $\theta$ (resp. $\bar{\theta}$ ) as the solution to the equation (2) with the boundary conditions

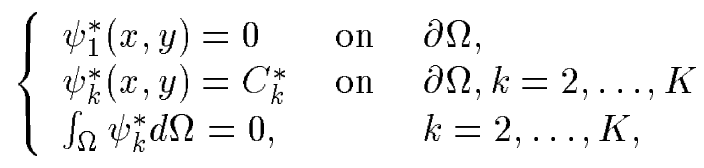

where $\psi^{*}$ is defined according to (5). We note (see e.g.[1]) that the problem (2), (8) has a unique solution in $\mathbf{H}^{\mathbf{1}}$ for any $\theta \in \mathbf{H}^{-\mathbf{1}}$ and that the norm $\|\cdot\|_{-1}$ is equivalent to the usual norm on $\mathbf{H}^{-\mathbf{1}}$ and

$$
a_{1}\|\nabla \psi\|_{\mathbf{L}^{2}} \leq\|\theta\|_{-1} \leq a_{2}\|\nabla \psi\|_{\mathbf{L}^{2}} .
$$

We recall some properties of the Jacobian operator in the following lemma [1],[2]. 
Lemma 2.1 The operator Jacobian verifies, for $u, v, w$ in $H_{0}^{1}(\Omega) \times H_{0}^{1}(\Omega) \times H_{0}^{1}(\Omega)$

$$
\begin{array}{r}
J(u, v)=-J(v, u), \quad \int_{\Omega} J(u, v) d \omega=0, \\
\int_{\Omega} J(u, v) w d \omega=\int_{\Omega} J(v, w) u d \omega, \\
\left|\int_{\Omega} J(u, v) \Delta u d \omega\right| \leq c_{4}\|v\|_{H^{2}}\|\nabla u\|_{L^{2}}\|\Delta u\|_{L^{2}} .
\end{array}
$$

These properties remain valid for functions $u, v, w$ constant on the boundary $\partial \Omega$.

From now on, we use the following notations :

$\|\cdot\|=\|\cdot\|_{L^{2}}$,

$\lambda_{1}$ is the first eigenvalue of the operator $-\Delta$ with Dirichlet boundary conditions,

$c_{1}$ is the constant of the Sobolev embedding $\|\psi\|_{L^{\infty}} \leq c_{1}\|\psi\|_{H^{2}}$,

$c_{2}$ is a constant such that $c_{2}\|\theta\|_{-1} \leq \sum H_{k}\left\|\Delta \psi_{k}\right\|$,

$c_{4}$ is a constant such that $\|\nabla \psi\|_{L^{4}}^{2} \leq c_{4}\|\nabla \psi\|\|\Delta \psi\|, \psi \in H_{0}^{1}(\Omega) \cap H^{2}(\Omega)$

$c_{\infty}$ is a constant such that $\|\psi\|_{H^{2}} \leq c_{\infty}\|\Delta \psi\|, \psi \in H_{0}^{1}(\Omega) \cap H^{2}(\Omega)$

$a_{1}$ and $a_{2}$ are constants such that

$a_{1}\|\nabla \psi\|_{\mathbf{L}^{2}} \leq\|\theta\|_{-1} \leq a_{2}\|\nabla \psi\|_{\mathbf{L}^{2}}$,

$H^{\prime}=\min _{k=1, \ldots, K} H_{k}$.

In this paper we rely on the following result proved in [2].

Theorem 2.1 For $\theta_{0, k} \in H^{-1}(\Omega), k=1, \ldots, K$, and $v \in L^{2}(\Omega)$ and for all $T>$ 0 , the system (1)- (7) has a unique solution $\theta$ in $\mathrm{C}\left([0, T], \mathbf{H}^{-\mathbf{1}}\right) \cap L^{2}\left(0, T, \mathbf{L}^{2}\right) \cap$ $L_{\text {loc }}^{2}\left(0, T, \mathbf{H}_{0}^{\mathbf{1}}\right)$. The function $\psi$ associated to $\theta$ by (2), (8) satisfies $\psi \in \mathrm{C}\left([0, T], \mathbf{H}^{\mathbf{1}}\right) \cap$ $L^{2}\left(0, T, \mathbf{H}^{\mathbf{2}}\right) \cap L_{\text {loc }}^{2}\left(0, T, \mathbf{H}^{\mathbf{3}}\right)$. The semi-group $G(t)$ from $\mathbf{H}^{-\mathbf{1}}$ in $\mathbf{H}^{-\mathbf{1}}, G(t) \theta_{0}(x)=$ $\theta(t, x)$, associated to these equations, is dissipative in the following sense:

$$
\begin{gathered}
\limsup _{t \rightarrow \infty}\|\theta(t)\|_{-1}^{2} \leq \frac{\|v\|^{2}}{\mu^{2} H_{1} \lambda_{1}^{2} c_{2}} \\
\limsup _{t \rightarrow \infty} \frac{1}{T} \int_{t}^{t+T} \sum H_{k}\left\|\Delta \psi_{k}\right\|^{2} d \tau \leq \frac{\|v\|^{2}}{\mu^{2} H_{1} \lambda_{1}^{2}}\left(1+\frac{1}{\mu T c_{2}}\right), \forall T>0 .
\end{gathered}
$$

Moreover there exists a global attractor $\mathcal{A}$ which is bounded in $\mathbf{L}^{2}$, compact and connected in $\mathbf{H}^{-1}$ and whose basin of attraction is the whole space $\mathbf{H}^{-\mathbf{1}}$. This attractor has finite Hausdorff and fractal dimensions in $\mathbf{H}^{-\mathbf{1}}$. 
In particular this theorem means that the long-time dynamics of the considered system is described by a finite-dimensional set in the phase space and therefore, at least theoretically, we may describe the long-time behaviour of solutions to (1)-(7) by a finite set of parameters.

\section{Statement of main results.}

As in [9]-[10], we will use the following

Definition 3.1 A finite set $\mathcal{L}=\left\{l_{j}\right\}_{1}^{M}$ of linearly independent bounded linear functionals on the space $\mathbf{H}^{2}=\left(H^{2}(\Omega)\right)^{K}$ is said to be a set of determining degrees of freedom for the problem (1)-(7) if for any two solutions $\theta^{1}(t)$ and $\theta^{2}(t)$ satisfying

$$
\lim _{t \rightarrow \infty} l_{j}\left(\psi^{1}(t)-\psi^{2}(t)\right)=0,1 \leq j \leq M
$$

where $\psi^{i}$ is defined from $\theta^{i}$ by (2) and (8), we have

$$
\lim _{t \rightarrow \infty}\left\|\theta^{1}(t)-\theta^{2}(t)\right\|_{-1}=0 .
$$

We note that, due to (9), the last inequality is equivalent to

$$
\lim _{t \rightarrow \infty}\left\|\psi^{1}(t)-\psi^{2}(t)\right\|_{\mathbf{H}^{1}}=0 .
$$

Our main results are the following assertions.

Theorem 3.1 Assume that the set $\mathcal{L}=\left\{l_{j}\right\}_{1}^{M}$ possesses the property

$$
\|\psi\|_{\mathbf{H}^{\mathbf{1}}} \leq C_{\mathcal{L}} \max _{j}\left|l_{j}(\psi)\right|+\varepsilon_{\mathcal{L}}\|\psi\|_{\mathbf{H}^{2}}
$$

for any $\psi \in \mathbf{H}^{2}$ with some positive constant $C_{\mathcal{L}}$ and $\varepsilon_{\mathcal{L}}$. Assume that

$$
\varepsilon_{\mathcal{L}}<\frac{a_{1}}{a_{2}} \frac{\lambda_{1} \mu^{2}}{\|v\|} \frac{\sqrt{H_{1} H^{\prime}}}{\sqrt{2} c_{\infty}^{2}} \min _{k=1, \ldots, K}\left(\sqrt{\frac{H_{k}}{\alpha_{k}}}\right) .
$$

where

$$
\alpha_{k}=2 c_{4}^{2} H_{k}+4 \frac{c_{1}^{2}}{\lambda_{1}}\left(\frac{p_{k-1}^{2}}{H_{k-1}}+\frac{p_{k}^{2}}{H_{k+1}}\right) .
$$

Then the set $\mathcal{L}$ is a set of determining degrees of freedom for the problem (1)-(7).

$\operatorname{RR} n^{\circ} 3067$ 
The importance of the relation (13) for construction of determining sets of functionals was first observed in [9] (see also [10]).

Theorem 3.1 implies

Corollary 3.1 Let $T^{h}$ be a triangulation of the domain $\Omega$ made of triangles with sides less than $h$ and let $\left\{x_{j}, j=1, \ldots, L_{h}\right\}$ be the set of all vertices of triangles from $T^{h}$. Then the set $\mathcal{L}=\left\{l_{j, k}(\psi)=\psi_{k}\left(x_{j}\right), j=1, \ldots, L_{h}, k=1, \ldots, K\right\}$ is a set of determining degrees of freedom for the problem (1)-(7) provided

$$
h<\frac{1}{C} \frac{a_{1}}{a_{2}} \frac{\lambda_{1} \mu^{2}}{\|v\|} \frac{\sqrt{H_{1} H^{\prime}}}{\sqrt{2} c_{\infty}^{2}} \min _{k=1, \ldots, K}\left(\sqrt{\frac{H_{k}}{\alpha_{k}}}\right),
$$

where $\alpha_{k}$ is defined by (15) and C is the constant of approximation (see (16) below).

This corollary follows from well-known estimate (see e.g. [8]) for linear splines connected with the triangulation $T^{h}$ of the smooth domain $\Omega$ :

$$
\left\|\omega-S\left(T^{h}, \omega\right)\right\|_{H^{1}} \leq C h\|\omega\|_{H^{2}},
$$

where $S\left(T^{h}, \omega\right)$ is the linear interpolation spline corresponding to $\omega \in H^{2}(\Omega)$.

Theorem 3.2 Assume that $l_{j} \in \mathcal{L}$ do not involve the last component of the vector of the stream functions $\psi_{1}, \ldots, \psi_{K}$, i.e. $l_{j}(\psi)$ is independent of $\psi_{K}$ for any $\psi \in \mathbf{H}^{2}$ and that the set $\mathcal{L}=\left\{l_{j}\right\}_{1}^{M}$ satisfies

$$
\sqrt{\sum_{k=1}^{K-1}\left\|\psi_{k}\right\|_{H^{1}}^{2}} \leq C_{\mathcal{L}} \max _{j}\left|l_{j}(\psi)\right|+\varepsilon_{\mathcal{L}} \sqrt{\sum_{k=1}^{K-1}\left\|\psi_{k}\right\|_{H^{2}}^{2}},
$$

for any $\psi \in \mathbf{H}^{2}$ with some positive constant $C_{\mathcal{L}}$ and $\varepsilon_{\mathcal{L}}$. Assume also that the bottom friction $\sigma$ satisfies

$$
\mu \lambda_{1}+2 \sigma \geq \frac{2\|v\|^{2} c_{\infty}^{2} a_{2}^{2}}{H_{K} H_{1} \mu^{3} \lambda_{1}^{2} a_{1}^{2}} \max _{k=1, \ldots, K}\left(\frac{\alpha_{k}}{H_{k}}\right)
$$

Then the set $\mathcal{L}$ is a set of determining degrees of freedom for the problem (1)-(7) provided

$$
\varepsilon_{\mathcal{L}}<\frac{a_{1}}{a_{2}} \frac{\lambda_{1} \mu^{2}}{\|v\|} \frac{\sqrt{H_{1} H^{\prime \prime}}}{\sqrt{2} c_{\infty}^{2}} \min _{k=1, \ldots, K}\left(\sqrt{\frac{H_{k}}{\alpha_{k}}}\right)
$$

where $\alpha_{k}$ is defined by (15) and $H "=\min _{k=1, \ldots, K-1} H_{k}$. 
From this theorem in the same way as corollary 3.1 , we have

Corollary 3.2 Assume $T^{h}$ and $x_{j}, j=1, \ldots, L_{h}$ as in corollary 3.1. Then the set $\mathcal{L}^{*}=l_{j, k}(\psi)=\psi_{k}\left(x_{j}\right), j=1, \ldots, L_{h}, k=1, \ldots, K-1$ is a set of determining degrees of freedom for the problem (1)-(7) provided (18) holds and

$$
h<\frac{1}{C} \frac{a_{1}}{a_{2}} \frac{\lambda_{1} \mu^{2}}{\|v\|} \frac{\sqrt{H_{1} H^{\prime \prime}}}{\sqrt{2} c_{\infty}^{2}} \min _{k=1, \ldots, K}\left(\sqrt{\frac{H_{k}}{\alpha_{k}}}\right),
$$

where $\alpha_{k}$ is defined by (15).

Theorem 3.2 and Corollary 3.2 mean that in order to determine completely asymptotical dynamics of solutions, we need no information about the behaviour of the K-th layer. A similar effect was first observed in [6] for some classes of reaction-diffusion systems. It was shown there that under some conditions certain non-vanishing components of a solution are not essential for description of long-time dynamics. We also refer to [5] for discussion of this phenomenon for the second order in time evolution equations.

Theorem 3.3 Assume that $\theta^{(j)}(t), j=1,2$ are two solutions belonging to the attractor. Assume also that either the condition (14) or conditions (18) and (19) are fulfilled. Let $\mathcal{L}$ be the corresponding set of determining degrees of freedom. Then the property

$$
l\left(\psi^{(1)}(t)\right)=l\left(\psi^{(2)}(t)\right)
$$

for any $l \in \mathcal{L}$ and $-\infty<t \leq t_{0}$ implies $\theta^{(1)}(t)=\theta^{(2)}(t)$ for $-\infty<t<\infty$.

A similar assertion on determining modes for the 2D Navier-Stokes equations was first obtain by Ladyzhenskaya in 1972 (see, e.g., references in [4], [19]).

As in [17],[18], we can also prove assertions similar to corollaries 3.1 and 3.2 and theorem 3.3 for local volume averages. In this case the determining functionals are defined by $l_{j}\left(\psi_{k}\right)=\frac{1}{h^{2}} \int_{\Omega} \psi_{k}(x, y) \lambda\left(\frac{x}{h}-j_{1}, \frac{y}{h}-j_{2}\right) d \omega$ where $\lambda(x, y)=1$ for $(x, y) \in S=[0,1] \times[0,1]$ and $\lambda(x, y)=0$ for $(x, y) \notin S$.

\section{Proofs.}

Proof of theorem 3.1. Consider $\tilde{\theta}$ and $\bar{\theta}$ two solutions of the system (1)-(6) with initial data $\tilde{\theta}_{0}$ and $\bar{\theta}_{0}$ respectively. We introduce $\theta=\tilde{\theta}-\bar{\theta}, \psi$ defined by $(2)$ and 
(8). Then $\theta$ satisfies the system

$$
\begin{aligned}
\frac{\partial \theta_{k}}{\partial t}-\mu \Delta^{2} \psi_{k}+D_{k}^{b o t t o m}=-J\left(\psi_{k}, \tilde{\theta}_{k}+\beta y\right)-J\left(\bar{\psi}_{k}, \theta_{k}\right) & \text { in } \quad \Omega, k=1, \ldots, \mathbb{l}(2) 1) \\
\theta=\Delta \psi-\mathcal{W} \psi & \text { in } \quad \Omega .
\end{aligned}
$$

Since $\psi_{k}^{*}(x, y, t)=C_{k}^{*}(t)$ on $\partial \Omega$ by $(6)$, let us introduce $C(t)=B C^{*}(t)$ and $\psi_{k}^{\prime}(x, y, t)=\psi_{k}(x, y, t)-C_{k}(t)$. Multiplying (21) by $H_{k} \psi_{k}^{\prime}$, integrating over $\Omega$ and summing on $k$, we obtain

$$
\frac{1}{2} \frac{d\|\theta\|_{-1}^{2}}{d t}+\mu \sum_{k} H_{k}\left\|\Delta \psi_{k}\right\|^{2}+H_{K} \sigma\left\|\nabla \psi_{K}\right\|^{2}=\sum_{k} H_{k}\left(J\left(\bar{\psi}_{k}, \theta_{k}\right), \psi_{k}^{\prime}\right) .
$$

Let us bound from above the right-hand side of this equation. We rewrite $\theta_{k}$ with its explicit form :

$$
\theta_{k}=\Delta \psi_{k}-\frac{p_{k}}{H_{k}}\left(\psi_{k}-\psi_{k+1}\right)-\frac{p_{k-1}}{H_{k}}\left(\psi_{k}-\psi_{k-1}\right) .
$$

Thus,

$$
\begin{gathered}
\sum_{k} H_{k}\left(J\left(\bar{\psi}_{k}, \theta_{k}\right), \psi_{k}^{\prime}\right)=\sum_{k} H_{k}\left(J\left(\bar{\psi}_{k}, \Delta \psi_{k}\right), \psi_{k}^{\prime}\right)- \\
-\sum_{k} p_{k}\left(J\left(\bar{\psi}_{k}, \psi_{k}-\psi_{k+1}\right), \psi_{k}^{\prime}\right)-\sum_{k} p_{k-1}\left(J\left(\bar{\psi}_{k}, \psi_{k}-\psi_{k-1}\right), \psi_{k}^{\prime}\right) .
\end{gathered}
$$

An integration by part and lemma 2.1 allow us to bound from above the first term of the right-hand side of the above equality by

$$
\left|\sum_{k} H_{k}\left(J\left(\bar{\psi}_{k}, \Delta \psi_{k}\right), \psi_{k}^{\prime}\right)\right| \leq c_{4} \sum_{k} H_{k}\left\|\bar{\psi}_{k}\right\|_{H^{2}}\left\|\nabla \psi_{k}\right\|\left\|\Delta \psi_{k}\right\| .
$$

On the other hand,

$$
\begin{gathered}
\left|\sum_{k} p_{k}\left(J\left(\bar{\psi}_{k}, \psi_{k}-\psi_{k+1}\right), \psi_{k}^{\prime}\right)\right|=\left|\sum_{k} p_{k}\left(J\left(\psi_{k}^{\prime}, \psi_{k+1}\right), \bar{\psi}_{k}\right)\right|= \\
\leq \sum_{k} p_{k}\left\|\bar{\psi}_{k}\right\|_{L^{\infty}}\left\|\nabla \psi_{k}\right\|\left\|\nabla \psi_{k+1}\right\|, \\
\left|\sum_{k} p_{k}\left(J\left(\bar{\psi}_{k}, \psi_{k}-\psi_{k+1}\right), \psi_{k}^{\prime}\right)\right| \leq \frac{c_{1}}{\sqrt{\lambda_{1}}} \sum_{k} p_{k}\left\|\bar{\psi}_{k}\right\|_{H^{2}}\left\|\nabla \psi_{k}\right\|\left\|\Delta \psi_{k+1}\right\| .
\end{gathered}
$$


Inserting the inequalities (25) and (24) in (23), we have

$$
\begin{gathered}
\frac{1}{2} \frac{d\|\theta\|_{-1}^{2}}{d t}+\mu \sum_{k} H_{k}\left\|\Delta \psi_{k}\right\|^{2}+H_{K} \sigma\left\|\nabla \psi_{K}\right\|^{2} \leq \\
\leq c_{4} \sum_{k} H_{k}\left\|\bar{\psi}_{k}\right\|_{H^{2}}\left\|\nabla \psi_{k}\right\|\left\|\Delta \psi_{k}\right\|+ \\
+\frac{c_{1}}{\sqrt{\lambda_{1}}} \sum_{k} p_{k}\left\|\bar{\psi}_{k}\right\|_{H^{2}}\left\|\nabla \psi_{k}\right\|\left\|\Delta \psi_{k+1}\right\|+ \\
+\frac{c_{1}}{\sqrt{\lambda_{1}}} \sum_{k} p_{k-1}\left\|\bar{\psi}_{k}\right\|_{H^{2}}\left\|\nabla \psi_{k}\right\|\left\|\Delta \psi_{k-1}\right\| \cdot \\
\frac{1}{2} \frac{d\|\theta\|_{-1}^{2}}{d t}+\mu \sum_{k} H_{k}\left\|\Delta \psi_{k}\right\|^{2}+H_{K} \sigma\left\|\nabla \psi_{K}\right\|^{2} \leq \\
\leq \sum_{k} \frac{\mu H_{k}}{4}\left\|\Delta \psi_{k}\right\|^{2}+\sum_{k} \frac{c_{4}^{2} H_{k}}{\mu}\left\|\bar{\psi}_{k}\right\|_{H^{2}}^{2}\left\|\nabla \psi_{k}\right\|^{2}+ \\
+\sum_{k} \frac{\mu H_{k-1}}{8}\left\|\Delta \psi_{k-1}\right\|^{2}+\sum_{k} \frac{2 c_{1}^{2} p_{k-1}^{2}}{\lambda_{1} \mu H_{k-1}}\left\|\bar{\psi}_{k}\right\|_{H^{2}}^{2}\left\|\nabla \psi_{k}\right\|^{2}+ \\
+\sum_{k} \frac{\mu H_{k+1}}{8}\left\|\Delta \psi_{k+1}\right\|^{2}+\sum_{k} \frac{2 c_{1}^{2} p_{k}^{2}}{\lambda_{1} \mu H_{k+1}}\left\|\bar{\psi}_{k}\right\|_{H^{2}}^{2}\left\|\nabla \psi_{k}\right\|^{2}
\end{gathered}
$$

Then

$\frac{d\|\theta\|_{-1}^{2}}{d t}+\mu \sum_{k} H_{k}\left\|\Delta \psi_{k}\right\|^{2}+2 H_{K} \sigma\left\|\nabla \psi_{K}\right\|^{2} \leq \frac{1}{\mu} \sum_{k} \alpha_{k}\left\|\bar{\psi}_{k}\right\|_{H^{2}}^{2}\left\|\nabla \psi_{k}\right\|^{2}$,

where $\alpha_{k}$ is defined by (15). Finally,

$$
\begin{gathered}
\frac{d\|\theta\|_{-1}^{2}}{d t}+\mu \sum_{k} H_{k}\left\|\Delta \psi_{k}\right\|^{2}+2 H_{K} \sigma\left\|\nabla \psi_{K}\right\|^{2} \leq \\
\leq \frac{c_{\infty}^{2}}{\mu a_{1}^{2}} \max _{k=1, \ldots, K}\left(\frac{\alpha_{k}}{H_{k}}\right) \sum_{k} H_{k}\left\|\Delta \bar{\psi}_{k}\right\|^{2}\|\theta\|_{-1}^{2} .
\end{gathered}
$$

Assuming that the family $\mathcal{L}$ fulfills (13), we have

$$
\|\nabla \psi\| \leq C_{\mathcal{L}} \max _{j}\left|l_{j}(\psi)\right|+\varepsilon_{\mathcal{L}} c_{\infty}\|\Delta \psi\| .
$$

RR $n^{\circ} 3067$ 
It is easy to see that

$$
\|\Delta \psi\|^{2} \geq \frac{1-\delta}{\varepsilon_{\mathcal{L}}^{2} c_{\infty}^{2}}\|\nabla \psi\|^{2}-C_{\delta, \mathcal{L}} \max _{j}\left|l_{j}(\psi)\right|^{2}
$$

for any positive $\delta$. Consequently

$$
\begin{aligned}
\sum_{k} H_{k}\left\|\Delta \psi_{k}\right\|^{2} & \geq \min _{k} H_{k} \sum_{k}\left\|\Delta \psi_{k}\right\|^{2} \\
& \geq \frac{1-\delta}{\varepsilon_{\mathcal{L}}^{2} c_{\infty}^{2}} H^{\prime}\|\nabla \psi\|^{2}-\tilde{C}_{\delta, \mathcal{L}} \max _{j}\left|l_{j}(\psi)\right|^{2}, \\
& \geq \frac{1-\delta}{\varepsilon_{\mathcal{L}}^{2} c_{\infty}^{2} a_{2}^{2}} H^{\prime}\|\theta\|_{-1}^{2}-\tilde{C}_{\delta, \mathcal{L}} \max _{j}\left|l_{j}(\psi)\right|^{2},
\end{aligned}
$$

for any positive $\delta$. Using this formula, the estimate (27), and the constants in (10), we obtain

$$
\begin{array}{r}
\frac{d\|\theta\|_{-1}^{2}}{d t}+\left\{\mu \frac{1-\delta}{\varepsilon_{\mathcal{L}}^{2} c_{\infty}^{2} a_{2}^{2}} H^{\prime}-\frac{c_{\infty}^{2}}{\mu a_{1}^{2}} \max _{k=1, \ldots, K}\left(\frac{\alpha_{k}}{H_{k}}\right) \sum_{k} H_{k}\left\|\Delta \bar{\psi}_{k}\right\|^{2}\right\}\|\theta(t)\|_{-1}^{2} \leq \\
\leq \mu \tilde{C}_{\delta, \mathcal{L}} \max _{j}\left|l_{j}(\psi)\right|^{2}
\end{array}
$$

for any positive $\delta$. We now proceed as in [18] and using [18, Lemma 3.1] along with formula (12) for $T=\mu^{-1} c_{2}^{-1}$, we obtain that $\|\theta(t)\|_{-1} \rightarrow 0$ as $t \rightarrow \infty$ provided

$$
\mu \frac{H^{\prime}}{c_{\infty}^{2} a_{2}^{2} \varepsilon_{\mathcal{L}}^{2}}-\frac{2\|v\|^{2} c_{\infty}^{2}}{\mu^{3} H_{1} \lambda_{1}^{2} a_{1}^{2}} \max _{k=1, \ldots, K}\left(\frac{\alpha_{k}}{H_{k}}\right)>0
$$

which we rewrite

$$
\varepsilon_{\mathcal{L}}<\frac{a_{1}}{a_{2}} \frac{\lambda_{1} \mu^{2}}{\|v\|} \frac{\sqrt{H_{1} H^{\prime}}}{\sqrt{2} c_{\infty}^{2}} \min _{k}\left(\sqrt{\frac{H_{k}}{\alpha_{k}}}\right) .
$$

Proof of theorem 3.2. We recall the estimate (26) of the proof of theorem 3.1 :

$$
\begin{aligned}
\frac{d\|\theta\|_{-1}^{2}}{d t}+\mu \sum_{k} H_{k} \| & \Delta \psi_{k}\left\|^{2}+2 H_{K} \sigma\right\| \nabla \psi_{K} \|^{2} \leq \\
& \leq \frac{1}{\mu} \sum_{k} \alpha_{k}\left\|\bar{\psi}_{k}\right\|_{H^{2}}^{2}\left\|\nabla \psi_{k}\right\|^{2}
\end{aligned}
$$


where $\alpha_{k}$ is defined by (15). Since the family $\mathcal{L}$ satisfies $(17)$, we have

$$
\sqrt{\sum_{k=1}^{K-1}\left\|\nabla \psi_{k}\right\|^{2}} \leq C_{\mathcal{L}} \max _{j}\left|l_{j}(\psi)\right|+\varepsilon_{\mathcal{L}} c_{\infty} \sqrt{\sum_{k=1}^{K-1}\left\|\Delta \psi_{k}\right\|^{2}} .
$$

We then obtain

$$
\begin{aligned}
\sum_{k=1, \ldots, K-1} H_{k}\left\|\Delta \psi_{k}\right\|^{2} & \geq \min _{k=1, \ldots, K-1} H_{k} \sum_{k=1, \ldots, K-1}\left\|\Delta \psi_{k}\right\|^{2} \\
& \geq \frac{1-\delta}{\varepsilon_{\mathcal{L}}^{2} c_{\infty}^{2}} H^{\prime \prime} \sum_{k=1}^{K-1}\left\|\nabla \psi_{k}\right\|^{2}-\tilde{C}_{\delta, \mathcal{L}} \max _{j}\left|l_{j}(\psi)\right|^{2},
\end{aligned}
$$

for any positive $\delta$. We then have

$$
\begin{aligned}
& \frac{d\|\theta\|_{-1}^{2}}{d t}+\mu \frac{1-\delta}{\varepsilon_{\mathcal{L}}^{2} c_{\infty}^{2}} H " \sum_{k=1}^{K-1}\left\|\nabla \psi_{k}\right\|^{2}+\left(\mu \lambda_{1}+2 \sigma\right) H_{K}\left\|\nabla \psi_{K}\right\|^{2} \leq \\
& \leq \frac{c_{\infty}^{2}}{\mu a_{1}^{2}} \max _{k=1, \ldots, K}\left(\frac{\alpha_{k}}{H_{k}}\right) \sum_{k=1}^{K} H_{k}\left\|\Delta \bar{\psi}_{k}\right\|^{2}\|\theta\|_{-1}^{2}+\mu \tilde{C}_{\delta, \mathcal{L}} \max _{j}\left|l_{j}(\psi)\right|^{2} .
\end{aligned}
$$

Let us introduce

$$
m\left(\varepsilon_{\mathcal{L}}, \sigma\right)=\min \left(\mu \frac{1-\delta}{\varepsilon_{\mathcal{L}}^{2} c_{\infty}^{2} a_{2}^{2}} H^{\prime \prime}, \frac{\mu \lambda_{1}+2 \sigma}{a_{2}^{2}} H_{K}\right) .
$$

The last inequality gives

$$
\begin{array}{r}
\frac{d\|\theta\|_{-1}^{2}}{d t}+\left(m\left(\varepsilon_{\mathcal{L}}, \sigma\right)-\frac{c_{\infty}^{2}}{\mu a_{1}^{2}} \max _{k=1, \ldots, K}\left(\frac{\alpha_{k}}{H_{k}}\right) \sum_{k=1}^{K} H_{k}\left\|\Delta \bar{\psi}_{k}\right\|^{2}\right)\|\theta\|_{-1}^{2} \leq \\
\leq \mu \tilde{C}_{\delta, \mathcal{L}} \max _{j}\left|l_{j}(\psi)\right|^{2} .
\end{array}
$$

We then proceed as in the proof of theorem 3.1 and obtain that, if

$$
\left(m\left(\varepsilon_{\mathcal{L}}, \sigma\right)-\frac{c_{\infty}^{2}}{\mu a_{1}^{2}} \max _{k=1, \ldots, K}\left(\frac{\alpha_{k}}{H_{k}}\right) \frac{2\|v\|^{2}}{\mu^{2} H_{1} \lambda_{1}^{2}}\right)>0
$$

i.e. if $\varepsilon_{\mathcal{L}}$ and $\sigma$ satisfy (18) and (19) then $\|\theta(t)\|_{-1}^{2} \rightarrow 0$ as $t \rightarrow \infty$.

If we assume that some layers (e.g., $k_{j}$ layers, $j=1, \ldots, s$ ) have large enough viscosities (the coefficients before $\Delta^{2} \psi$ in the main equation), then using similar method, 
we can prove that the behaviour of these layers is completely determined by other (essential) layers.

Proof of theorem 3.3. Rewriting in an uniform way the inequalities (28) and (29), for both cases we have, with $\theta=\theta^{(1)}-\theta^{(2)}$,

$$
\frac{d\|\theta\|_{-1}^{2}}{d t}+a(t)\|\theta\|_{-1}^{2} \leq C C_{\mathcal{L}}^{2} \max _{j}\left|l_{j}(\psi)\right|^{2} \quad \forall t \in \mathbb{R}
$$

with

$$
a(t)=\left\{\mu \frac{1-\delta}{\varepsilon_{\mathcal{L}}^{2} c_{\infty}^{2} a_{2}^{2}} H^{\prime}-\frac{c_{\infty}^{2}}{\mu a_{1}^{2}} \max _{k=1, \ldots, K}\left(\frac{\alpha_{k}}{H_{k}}\right) \sum_{k} H_{k}\left\|\Delta \psi_{k}^{2}\right\|^{2}\right\}
$$

in the first case, and

$$
a(t)=\left\{m\left(\varepsilon_{\mathcal{L}}, \sigma\right)-\frac{c_{\infty}^{2}}{\mu a_{1}^{2}} \max _{k=1, \ldots, K}\left(\frac{\alpha_{k}}{H_{k}}\right) \sum_{k=1}^{K} H_{k}\left\|\Delta \psi_{k}^{2}\right\|^{2}\right\}
$$

with

$$
m\left(\varepsilon_{\mathcal{L}}, \sigma\right)=\min \left(\mu \frac{1-\delta}{\varepsilon_{\mathcal{L}}^{2} c_{\infty}^{2} a_{2}^{2}} H^{\prime \prime}, \frac{\mu \lambda_{1}+2 \sigma}{a_{2}^{2}} H_{K}\right)
$$

in the second one. If condition (20) is verified, we have

$$
\frac{d\|\theta\|_{-1}^{2}}{d t}+a(t)\|\theta\|_{-1}^{2} \leq 0 \quad \forall t \leq t_{0}
$$

Integrating this last inequality between $s$ and $t$, we obtain

$$
\|\theta(t)\|_{-1}^{2} \leq\|\theta(s)\|_{-1}^{2} \exp \left(-\int_{s}^{t} a(\tau) d \tau\right)
$$

Consider now that $\theta^{(i)}$ belongs on the attractor. We then have for all $t \in \mathbb{R}$

$$
\begin{gathered}
\left\|\theta^{(i)}(t)\right\|_{-1}^{2} \leq \frac{\|v\|^{2}}{\mu^{2} H_{1} \lambda_{1}^{2} c_{2}} \\
\frac{1}{T} \int_{t}^{t+T} \sum H_{k}\left\|\Delta \psi_{k}^{(i)}\right\|^{2} d \tau \leq \frac{\|v\|^{2}}{\mu^{2} H_{1} \lambda_{1}^{2}}\left(1+\frac{1}{\mu T c_{2}}\right), \forall T>0 .
\end{gathered}
$$


Moreover, by conditions (14) or (18) - (19), we have

$$
a(t)>\frac{c_{\infty}^{2}}{\mu a_{1}^{2}} \max _{k=1, \ldots, K}\left(\frac{\alpha_{k}}{H_{k}}\right)\left[\frac{2\|v\|^{2}}{\mu^{2} H_{1} \lambda_{1}^{2}}-\sum_{k=1}^{K} H_{k}\left\|\Delta \psi_{k}^{2}\right\|^{2}\right] .
$$

Combining the above inequality with (32),

$$
\int_{s}^{t} a(\tau) d \tau>\frac{c_{\infty}^{2}}{\mu a_{1}^{2}} \max _{k=1, \ldots, K}\left(\frac{\alpha_{k}}{H_{k}}\right)\left[\frac{2\|v\|^{2}}{\mu^{2} H_{1} \lambda_{1}^{2}}(t-s)-\frac{\|v\|^{2}}{\mu^{2} H_{1} \lambda_{1}^{2}}\left((t-s)+\frac{1}{\mu c_{2}}\right)\right],
$$

and then

$$
\int_{s}^{t} a(\tau) d \tau>\frac{c_{\infty}^{2}}{\mu a_{1}^{2}} \max _{k=1, \ldots, K}\left(\frac{\alpha_{k}}{H_{k}}\right) \frac{\|v\|^{2}}{\mu^{2} H_{1} \lambda_{1}^{2}}\left[1-\frac{1}{\mu(t-s) c_{2}}\right](t-s) .
$$

Thus, for $s<t-\frac{2}{\mu c_{2}}$,

$$
\int_{s}^{t} a(\tau) d \tau>\frac{c_{\infty}^{2}}{\mu a_{1}^{2}} \max _{k=1, \ldots, K}\left(\frac{\alpha_{k}}{H_{k}}\right) \frac{\|v\|^{2}}{\mu^{2} H_{1} \lambda_{1}^{2}} \frac{1}{2}(t-s)=A(t-s)
$$

for any $-\infty<s \leq t-\frac{2}{\mu c_{2}}<t<t_{0}$. Combining (30) with (31) and (33) gives

$$
\|\theta(t)\|_{-1}^{2} \leq C \exp (-A(t-s)) .
$$

Now if we let $s \rightarrow-\infty$ we obtain $\|\theta(t)\|_{-1}=0 \quad \forall t \leq t_{0}$. We thus obtain $\theta^{(1)}(t)=\theta^{(2)}(t)$ for $t \leq t_{0}$. The uniqueness theorem for the system (1),(2) yields that $\theta^{(1)}(t)=\theta^{(2)}(t)$ for $t \in \mathbb{R}$.

\section{Acknowledgments.}

The authors would like to thank Professor M. Pierre for very stimulating discussions on the subject. This paper was written while I.C. was visiting the Institut Elie Cartan (Nancy 1). He would like to thank people at this institution for their very kind hospitality. He is especially grateful to Professor P.A. Vuillermot. Thanks to his efforts this visit became possible and was very fruitful. The University Henri-PoincaréNancy 1 and the French-Russian Lyapunov Center are also highly acknowledged for their financial support. 


\section{References}

[1] C. Bernier Etude et parallélisation d'un code d'éléments finis pour la modélisation quasi-géostrophique des circulations océaniques. PhD thesis of INPG (Grenoble, France) (1990).

[2] C. Bernier Existence of attractor for the quasi-geostrophic approximation of the Navier-Stokes equations and estimate of its dimension. Advances in Mathematical Sciences and Applications, 4 (2), pp 465-489 (1994).

[3] K. Bryan, M.D. Cox A numerical investigation of the oceanic general circulation. Tellus, 19, pp 54-80 (1967).

[4] I.D. Chueshov Global attractors for non-linear problems of mathematical physics. Russian Math. Surveys, 48 (3), pp 133-161 (1993).

[5] I.D. Chueshov On the finiteness of the number of determining elements for von Karman evolution equations. to appear in Math. Methods in Appl. Sci.

[6] I.D. Chueshov Remark on sets of determining elements for reaction-diffusion systems. submitted to Math. Notes.

[7] I.D. Chueshov, E.S. Titi (to be submitted)

[8] P. Ciarlet The Finite Element Method for elliptic Problems. North-Holland, Amsterdam-New-York-Oxford, (1978).

[9] B. Cockburn, D.A. Jones, E.S. Titi Determining degrees of freedom for nonlinear dissipative systems. C. R. Acad. Sci. Paris, ser. 1, 321, pp 563-568 (1995).

[10] B. Cockburn, D.A. Jones, E.S. Titi Estimating the numbers of asymptotic degrees of freedom for nonlinear dissipative systems. submitted to Math. Comput.

[11] G.J. Fix Finite elements models for ocean circulation problems. SIAM J. Appl. Math., 29 (3), pp 371-387 (1975).

[12] C. Foias, I. Kukavica Determining nodes for the Kuramoto-Sivashinsky equation. J. Dynamics Diff. Eq., 7 (2), pp 365-373 (1995).

[13] C. Foias, G. Prodi Sur le comportement global des solutions non stationnaires des équations de Navier-Stokes en dimension deux. Rend. Sem. Mat. Univ. Padova, 36, pp 1-34 (1967). 
[14] C. Foias, R. Temam Determination of solutions of the Navier-Stokes equations by a set of nodal values. Math. Comput., 43, pp 117-133 (1984).

[15] C. Foias, E.S. Titi Determining nodes, finite difference schemes and inertial manifolds. Nonlinearity, 4, pp 135-153 (1991).

[16] W.R. Holland The role of mesoscale eddies in the general circulation of the ocean - numerical experiments using wind driven quasi-geostrophic model. Journal of Physical Oceanography, 8 (3), (1978).

[17] D.A. Jones, E.S. Titi Determination of the solutions of the Navier-Stokes equations by finite volume elements. Physica D, 60, pp 165-174 (1992).

[18] D.A. Jones, E.S. Titi Upper bounds on the number of determining modes, nodes and volume elements for the Navier-Stokes equations. Indiana Univ. Math. J., 42, pp 875-887 (1993).

[19] O.A. Ladyzhenskaya On the determination of minimal global attractors for the Navier-Stokes and other partial differential equations. Russian Math. Surveys, 42 (6), pp 27-73 (1987).

[20] C. Le Provost, C. Bernier, E. Blayo A comparison of two numerical methods for integrating a quasi geostrophic multilayer model of ocean circulations: finite element and finite difference methods. Journal of Computational Physics, 110 (2), pp 341-359 (1994) .

[21] J-L. Lions, R. Temam, S. Wang On the equations of large-scale ocean. Nonlinearity, 5, pp 1007-1053 (1992).

[22] J-L. Lions, R. Temam, S. Wang Mathematical theory for the coupled atmosphere-ocean models. J. Math. Pures et Appl., 74, pp 105-163 (1995).

[23] A.S. Sarkisyan On the dynamics of the origin of the wind driven currents in the baroclinic ocean. Okeanologie, 11, pp 393-409 (1962). 
Unit’e de recherche INRIA Lorraine, Technop^ole de Nancy-Brabois, Campus scientifique, 615 rue du Jardin Botanique, BP 101, 54600 VILLERS LÈS NANCY

Unit'e de recherche INRIA Rennes, Irisa, Campus universitaire de Beaulieu, 35042 RENNES Cedex Unit 'e de recherche INRIA Rh`one-Alpes, 655, avenue de l'Europe, 38330 MONTBONNOT ST MARTIN Unit'e de recherche INRIA Rocquencourt, Domaine de Voluceau, Rocquencourt, BP 105, 78153 LE CHESNAY Cedex

Unit’e de recherche INRIA Sophia-Antipolis, 2004 route des Lucioles, BP 93, 06902 SOPHIA-ANTIPOLIS Cedex

Éditeur

INRIA, Domaine de Voluceau, Rocquencourt, BP 105, 78153 LE CHESNAY Cedex (France)

ISSN 0249-6399 